2. - On the Lerch zeta function, Pacific J. Math 1 (1951), 161-167.

3. - Mathematical analysis, Addison-Wesley, Reading, Mass., 1957.

4. Shô Iseki, The transformation formula for the Dedekind modular function and related functional equations, Duke Math. J. 24 (1957), 653-662.

5. - A proof of a functional equation related to the theory of partitions, Proc. Amer. Math. Soc. 12 (1961), 502-505.

6. H. Rademacher, Zur Theorie der Modulfunktionen, J. Reine Angew. Math. 167 (1932), 312-336.

California Institute of Technology

\title{
ON THE HOMOTOPY CLASSES OF SELF-MAPPINGS OF BORDERED RIEMANN SURFACES
}

\section{CLIFFORD J. EARLE ${ }^{1}$}

1. Let $W$ be a Riemann surface which can be represented as the interior of a bordered surface $W_{0}$ with border $B$. Let $F: W \rightarrow W$ be a mapping which has continuous boundary values in $B$. Then $F$ can be extended by symmetry to a self-mapping $F_{*}$ of the doubled surface $W_{*}$. Let the given mapping $F$ be homotopic to the identity map of $W$ onto itself. It is natural to ask whether $F_{*}$ is homotopic to the identity also. In this note we give an affirmative answer for a large number of cases.

2. To be specific we assume that the universal covering surface of $W$ is the disk $U=\{z:|z|<1\} . W$ is of the form $W=U / G$ where $G$ is a Fuchsian group of linear transformations on $U$. $G$ is a group of the second kind; that is, the region of discontinuity $\Omega$ of $G$ contains points on the circumference $\partial U$ of $U . W$ is thus the interior of the bordered surface $W_{0}$ whose border $B$ corresponds to $\Omega \cap \partial U$. The double of $W_{0}$ is the surface $W_{*}=\Omega / G$. We assume that $G$ has at least three limit points, so that the universal covering surface of $W_{*}$ and $\Omega$ is the disk.

It is well known [1, pp. 98-99] that every mapping $F: W \rightarrow W$ is induced by a mapping $f: U \rightarrow U$. That is, $F \phi=\phi f$ where $\phi: U \rightarrow W$ is the natural projection. Moreover, $F$ is homotopic to the identity mapping of $W$ onto $W$ if and only if $f$ can be so chosen that $A f=f A$ for all $A$ in $G$.

Received by the editors March 25, 1963.

1 This research was supported in part by the Air Force Office of Scientific Research. 
TheOREM 1. Let $F: W \rightarrow W$ be homotopic to the identity and let $f: U \rightarrow U$ induce $F$ and satisfy $A f=f A$ for all $A$ in $G$. The following are equivalent:

1. $F$ has a continuous symmetric extension $F_{*}$ to $W_{*}$ which is homotopic to the identity under a homotopy which maps $B$ into itself at each stage.

2. $f$ is continuous on $\Omega \cap \partial U$ and maps each arc of $\Omega \cap \partial U$ into itself.

Proof. It is clear that 1 implies 2 . Indeed, restricting each mapping in the given homotopy to $W$ we obtain a family of mappings $F_{t}: W \rightarrow W_{*}$ such that $F_{0}$ is the identity and $F_{1}=F . F_{t}$ is induced by $f_{t}: U \rightarrow \Omega$. The $f_{t}$ can be chosen so that $f_{t}$ is continuous in $z$ and $t$, and $A f_{t}=f_{t} A$ for all $A$ in $G$ [1, pp. 99-100]. With this choice of $f_{t}, f_{0}$ is the identity and $f_{1}=f$. Since $F_{t}$ is continuous on $B$ and maps $B$ into itself, $f_{t}$ is continuous on $\Omega \cap \partial U$ and maps each arc $\alpha$ of $\Omega \cap \partial U$ into $\Omega \cap \partial U$. Since $f_{0}$ is the identity, $f_{0}(\alpha)=\alpha$. Therefore $f_{t}(\alpha) \subset \alpha$ for all $t$, in particular for $t=1$ as required.

Conversely let $f$ satisfying condition 2 be given. We extend $f$ to all of $\Omega$ by defining $f(1 / \bar{z})=1 / \bar{f}(z)$ for $z$ in $U$. By condition $2, f$ is continuous on $\Omega$. Moreover $f$ induces the self-mapping $F_{*}$ of $W_{*}$. We shall show that $F_{*}$ is homotopic to the identity by defining mappings $f_{t}: \Omega \rightarrow \Omega$ which induce the homotopy.

Following a suggestion of Professor Ahlfors we introduce the hyperbolic metric on $\Omega$. (This is possible because the universal covering surface of $\Omega$ is $U$.) Since this metric has negative curvature, for any $z$ in $\Omega$ there is a unique geodesic joining $z$ to $f(z)$. For $z$ in $U$ (ext $U$ ) this geodesic lies wholly in $U$ (ext $U$ ), and for $z$ in $\partial U$ the required geodesic is the arc joining $z$ to $f(z)$ in $\Omega \cap \partial U$. We define $f_{t}(z)$ to be that point on the geodesic from $z$ to $f(z)$ whose distances from $z$ and $f(z)$ stand in the proportion $t: 1-t$. Obviously $f_{t}$ is continuous in $z$ and $t$, $f_{0}$ is the identity, and $f_{1}=f$. Moreover, $f_{t}$ maps each arc of $\Omega \cap \partial U$ into itself.

We must show that $f_{t}$ induces a mapping $F_{t}: W_{*} \rightarrow W_{*}$. It suffices to show that $f_{t} A=A f_{t}$ for all $A$ in $G$. But $f_{t}(A(z))$ is a point on the geodesic from $A(z)$ to $f(A(z))=A(f(z))$, and this geodesic is the image under the isometry $A$ of the geodesic from $z$ to $f(z)$. Q.E.D.

3. From Theorem 1 we can obtain several conditions which imply that the extended mapping $F_{*}$ is homotopic to the identity. For instance, suppose $f$ is continuous at each point of $\partial U$. Then it follows from the equation $A f=f A$ for $A$ in $G$ that $f$ leaves each limit point of $G$ fixed. In particular, if $\alpha$ is an arc of $\Omega \cap \partial U$, each endpoint of $\alpha$ is left fixed, so that $f$ satisfies condition 2 . 
Among the mappings $f: U \rightarrow U$ which have continuous extensions to $\partial U$ are the quasiconformal mappings of $U$ onto itself. This proves

THEOREM 2. Let $F: W \rightarrow W$ be a quasiconformal homeomorphism which is homotopic to the identity. $F$ has a continuous symmetric extension $F_{*}$ to the double of $W . F_{*}$ is homotopic to the identity under a homotopy leaving each border contour of $W$ fixed.

For another example let the border contours of $W_{0}$ be compact and let $F$ map each contour of $B$ into itself. It follows that $f$ is continuous on $\partial U \cap \Omega$ and that $f$ maps an arc $\alpha$ of $\partial U \cap \Omega$ into some equivalent $\operatorname{arc} T(\alpha), T$ in $G$. We claim that $T(\alpha)=\alpha$. It suffices to find one common endpoint. Since $\alpha$ lies over a compact contour of $B$, there is a hyperbolic transformation $A$ in $G$ with $A(\alpha)=\alpha$. Consider a fixed $z$ in $\alpha, f(z)$ in $T(\alpha)$. As $n \rightarrow \infty, A^{n} f(z)$ approaches a fixpoint $\zeta$ of $A$. But $A^{n} f(z)=f\left(A^{n}(z)\right) \in T(\alpha)$, so $\zeta$ is an endpoint of $T(\alpha)$. Since $A^{n} z \rightarrow \zeta$, $\zeta$ is an endpoint of $\alpha$ as claimed.

THEOREM 3. Let $W_{0}=W \cup B$ be a bordered Riemann surface with interior $W$ and border $B$ consisting of compact contours. Let $F: W \rightarrow W$ have a continuous extension mapping each contour of $B$ into itself. Let $F_{*}$ be the symmetric extension of $F$ to the double. Let $F$ be homotopic to the identity. Then $F_{*}$ is homotopic to the identity under a homotopy leaving each contour of $B$ fixed.

\section{REFERENCE}

1. L. Bers, Analytic functions, Princeton Univ. Press, Princeton, N. J., 1960; pp. 89-119.

HARVARD UNIVERSITY 